EESTI NSV TEADUSTE AKADEEMIA TOIMETISED. XV KOIDE FOOSIKA MATEMAATIKA- JA TEHNIKATEADUSTE SEERIA. 1966, NR. 4

ИЗВЕСТИЯ АКАДЕМИИ НАУК ЭСТОНСКОИ ССР. ТОМ ХV СЕРИЯ ФИЗИКО-МАТЕМАТИЧЕСКИХ И ТЕХНИЧЕСКИХ НАУК. 1966, № 4

\title{
В. ПЫЛДМАА
}

\section{О РОЛИ КРАТНОГО РАССЕЯНИЯ В СУМЕРКАХ}

В последнее время интенсивно развивается спутниковая метеорология. Несмотря на это, не потеряло своего значения и исследование свойств земной атмосферы сумеречными методами. Некоторые задачи, решаемые методами сумеречного зондирования атмосферы, стали весьма актуальными именно в настоящее время, когда большие высоты стали доступными непосредственному исследованию. Изучение распределения и изменения яркостей сумеречного неба позволяет определить целый ряд параметров, характеризуюших физические свойства атмосферы.

В данной статье рассматриваются вопросы, касающиеся определения доли кратного рассеяния в сумерках, а также спектральных изменений яркостей неба.

\section{Кратное рассеяние в сумерках}

Определение роли кратного рассеяния в сумерках является целью мпогих исследований уже в течение нескольких десятков лет. Создан ряд моделей атмосферы и схем вычислений яркостей вторичного рассеяния $I_{2}$. Однако полученные результаты варьируются в очень широких пределах в зависимости от выбранной модели и исходных параметров вычислений.

Наряду с чисто теоретическими методами определения величины $I_{2}$ предложены и методы, опирающиеся на наблюдаемые яркости сумеречного неба. Широкое применение нашел метод В. Фесенкова [1, 2], который позволяет определить яркость $I_{2}$ на меридиане Солнца невысоко над горизонтом при достаточно больших зенитных расстояниях Солнца. В основе идеи В. Фесенкова лежит предположение, что в точках противо. вертикала Солнца, расположенных невысоко над горизонтом $\left(z \approx-70^{\circ}\right)$, при больших погружениях Солнца выполняется условие

$$
I_{2}(-z, \zeta, \lambda) \approx I(-z, \zeta, \lambda)
$$

( $z$ считается положительным на вертикале и отрицательным на противовертикале Солнца). Соответственно яркость кратного рассеяния в аналогичной точке вертикала Солнца определяется выражением

$$
I_{2}(+z, \zeta, \lambda)=k(\zeta, z) I_{2}(-z, \zeta, \lambda) \text {. }
$$

В последнее время предложены некоторые уточнения к упомянутому методу $[3,4]$, касающиеся определения яркостей $I_{2}$ на противовертиқале Солнца. А именно, вместо (1) рекомендовано выражение 


$$
I_{2}(\zeta,-z, \lambda) \cong I(\zeta,-z, \lambda)-\left[f(\zeta,-z, \lambda) / f\left(\zeta^{\prime},+z, \lambda\right)\right] I\left(\zeta^{\prime},+z, \lambda\right),
$$

где $f(\zeta, z, \lambda)$ - индикатриса рассеяния. $\zeta^{\prime}$ определяется из условия:

$$
I_{1}(\zeta,-z, \lambda)=\left[f(\zeta,-z, \lambda) / f\left(\zeta^{\prime},+z, \lambda\right)\right] I_{1}\left(\zeta^{\prime},+z, \lambda\right) .
$$

Вєпрос о границах применения этого метода подробно рассмотрен в $\left[{ }^{4}\right]$.
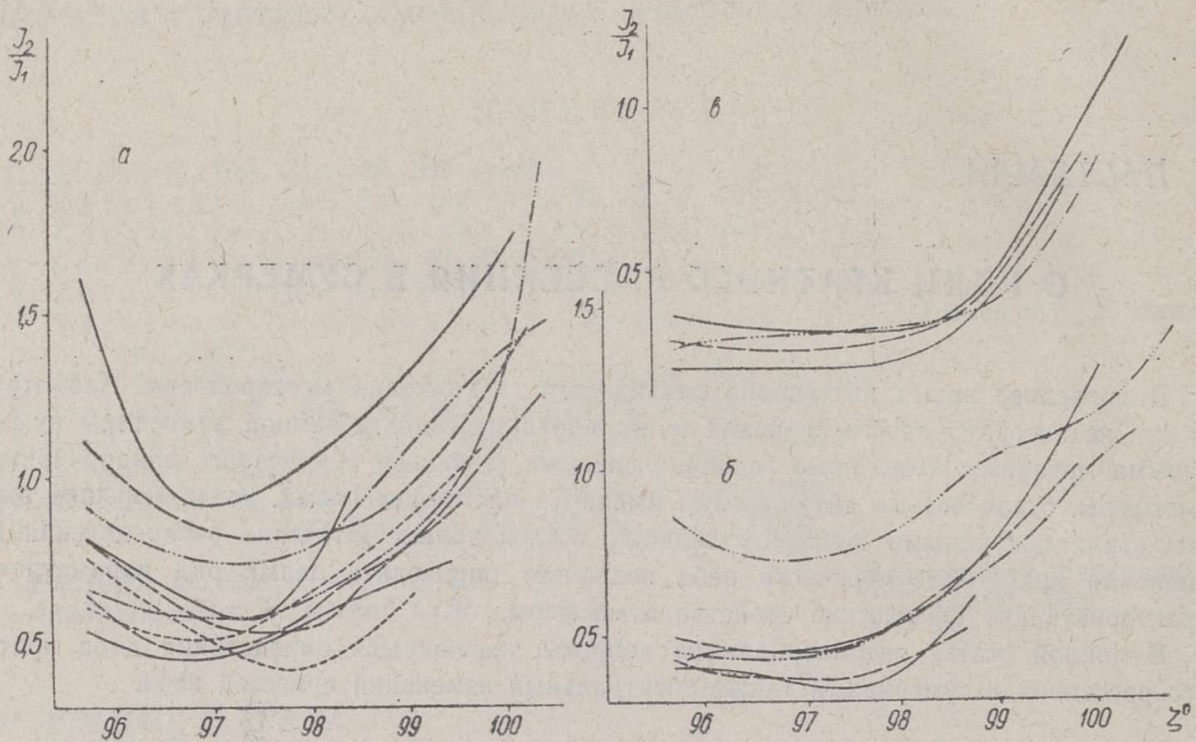

Рис. 1. Отношение $I_{2} / I_{1}$ по формулам (3) и (2) в зависимости от зенитного расстояния Солнца для направления $z=+70^{\circ}$ в разные числа: $a-\lambda=422$ мнк, б- $\lambda=479$ млк, в- $\lambda=574$ мик.
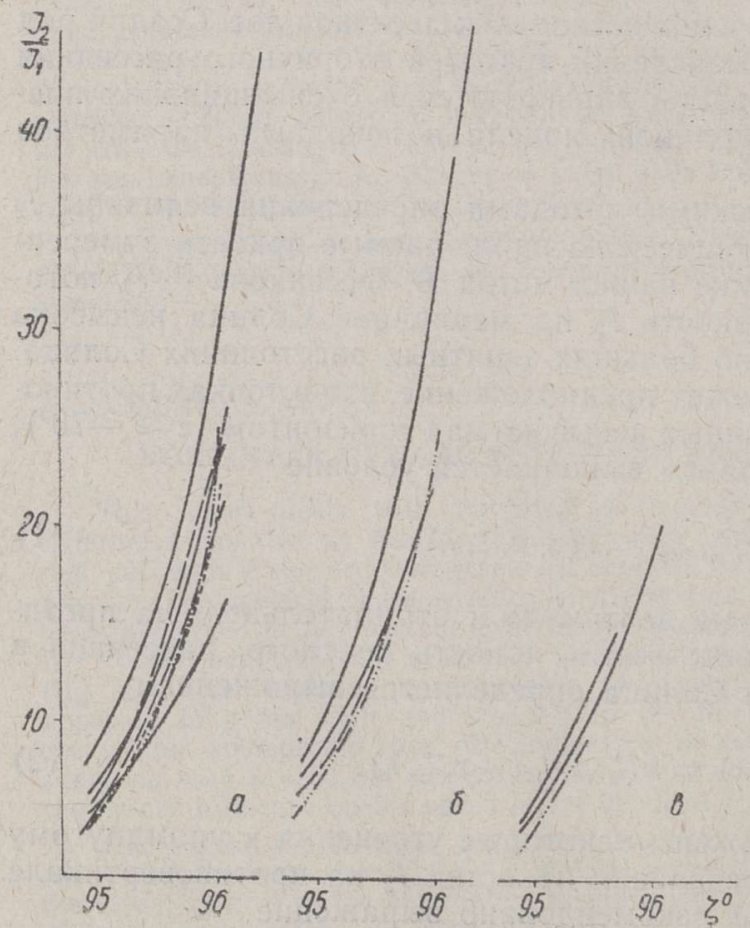

На основе измеренных яркостей сумеречного неба [5] автором определено по формулам (3) и (2) отношение $I_{2} / I_{1}$ при $z= \pm 70^{\circ}$ для ряда сумерек. На рис. 1 и 2 представлены полученные результаты. Обрашает на себя внимание относительно малый разброс значений $I_{2} / I_{1}$ для разных чисел (экспериментальные данные относятся к периоду с июня по сентябрь 1963 г.).

Сравнение величин $I_{2} / I_{1}$ в разных спектральных областях показывало, что доля кратного рассеяния растет в направлении коротких волн.

Рис. 2. Отношение $I_{2} / I_{1}$ по формуле (3) в зависимости от зенитного расстояния Солнца для направления $z=-70^{\circ}$ в разные числа: $a-\lambda=422$ мик, $\sigma-\lambda=$ $=479$ ммк, в- $\lambda=574$ ммк. 

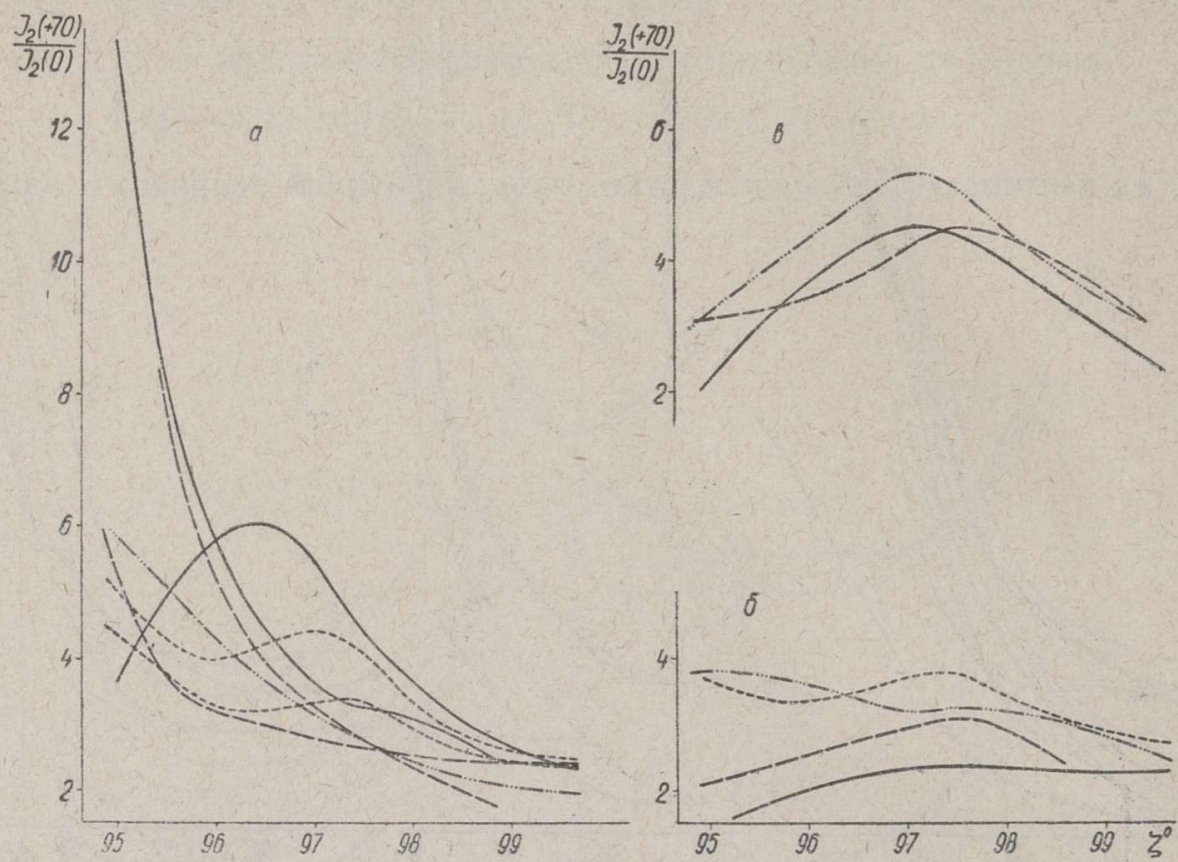

Рис. 4. Относительные изменения яркостей $I_{2}$ в направлениях $z=+70$ и $0^{\circ}$ в за. висимости от Ђ в разные числа: $a-\lambda=422$ ммк, $\sigma-\lambda=479$ мик, $B-\lambda=574$ млк.

Сравнение яркостей кратного рассєяния, полученных для разных направлений визирования, показывает большую изменчивость $I_{2}$ по меридиану Солнца. Судя по значениям $I_{2}$ для трех направлений $(z= \pm 70$ и $0^{\circ}$ ), можно сказать, что при любом определенном значении $\zeta$ в рассмотренной нами фазе сумерек яркость кратного рассеяния увеличивается к горизонту по обе стороны. При этом $I_{2}(+70)>I_{2}(-70)$. Это вполне понятно, если учитывать, что угол рассеяния ф для яркости $I_{2}$ при $z<0$ больше $\pi / 2$, но при $z>0 \varphi<\pi / 2\left[{ }^{4}\right]$. Кроме индикатрисы на $I_{2}=I_{2}(z)$ должна иметь влияние еще разность в оптических толщинах, особенно до акта вторичного рассеяния.

На рис. 4 представлены результаты сравнения яркостей $I_{2}$ в двух направлениях визирования $\left(z=+70\right.$ и $\left.0^{\circ}\right)$. При зенитных расстояниях Сюлнца $\zeta \approx 95 \div 97^{\circ}$ отсутствует единая определенная форма для функции $I_{2}(+70) / I_{2}(0)=g(\xi)$. Зато при значениях $\zeta \gtrsim 97^{\circ}$ с ростом глубины Солнца всегда наблюдается тенденция к выравниванию яркостей $I_{2}$ в рассмотренных направлениях.

Исходя из наших экспериментальных данных, яркость кратного рассеяния в направлении $z=-70^{\circ}$ оказалось возможным определить лишь для промежутка времени $\zeta \approx 95^{\circ} \div 96^{\circ} 20^{\prime}$. Поэтому сделать какие-нибудь общие заключения о ходе $I_{2}(z)=f(z)$ на противовертикале Солнца было невозможно. Однако и в этой фазе сумерек можно заметить убывание отношения $I_{2}(-70) / I_{2}(0)$ с ростом зенитного расстояния Солнца, причем яркость $I_{2}(-70)$ превышаєт яркость $I_{2}(0)$ в среднем в $1,5-2,5$ раза. Следовательно, увеличением зенитного расстояния Солнца (по крайней мере до $\zeta \approx 100^{\circ}$ ) распределение яркостей $I_{2}$ по меридиану Солнца выравнивается. Однако в недавно опубликованных результатах вычислений яркостей кратного рассеяния $I_{2}(z)$ Н. Дивари и Л. Плотни- 
ковой для определенной модели атмосферы [ $\left.{ }^{6}\right]$ такие изменения не наблюдаются - рассмотренные отношения остаются почти постоянными.

При интерпретации данных сумеречного зондирования атмосферы представляет интерес определение отношения яркостей кратного и первичного рассеяний $I_{2} / I_{1}$. Полученные автором значения $I_{2} / I_{1}$ для трех направлений визирования свидетельствуют о значительной изменчивости вдоль меридиана Солнца и этой величины. Так как отнсшения $I_{2} / I_{1}$, полученные для различных чисел, дали хорошее совпадение, нетрудно было найти их средние значения. На основе последних сравнивались отношения $I_{2} / I_{1}$ в направлениях $z= \pm 70$ и $0^{\circ}$. Полученные результаты приведены на рис. 5. Обра-

Рис. 5. Относительные изменения отношения $I_{2} / I_{1}$ в зависимости от зенитного расстояния Солнца, $B_{2}=\left[I_{2}(z) / I_{1}(z)\right]\left[I_{2}(0) / I_{1}(0)\right]^{-1}$ :

$$
\begin{gathered}
1-\lambda=422 \text { ммк, } 2-\lambda=479 \text { ммк, } \\
3-\lambda=574 \text { ммк; } a-z=-70^{\circ}, \\
\quad \sigma-z=+70^{\circ} .
\end{gathered}
$$
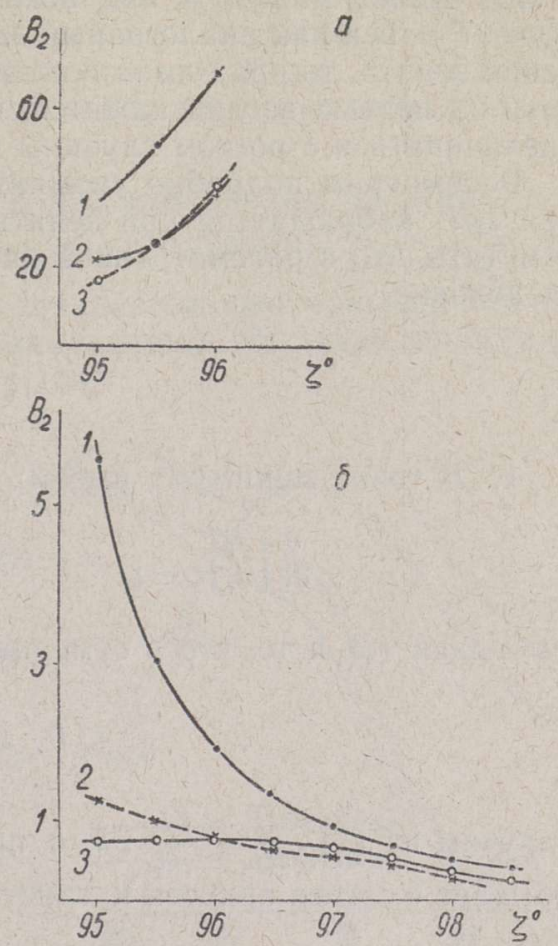

щает на себя внимание, что в рассматриваемой фазе сумерек рост глубины Солнца приносит с собой увеличение контрастов в распределении величин $I_{2} / I_{1}$ по меридиану Солнца. Нсключением в данном случае являются яркости для $\lambda=422 л м \kappa$ в направлениях $z=+70$ и $0^{\circ}$, где тенденция роста контрастов замечается несколько позже.

Имея в виду вышеизложенные результаты, можно заключить, что применение полученной для некоторого определенного направления визирования величины $I_{2}$ или $I_{2} / I_{1}$ непосредственно для других $z$ не оправдано. Переход от одного направления к другому должен быть опреде: лен на основе поведения функции $I_{2}(z)$. Но последняя является пока еще малоизученной.

Некоторые выводы о роли кратного рассеяния можно сделать по динамике яркостной картины сумеречного неба [']. Она, например, позволяет в первом приближении определить те значения зенитного расстояния Солнца, при которых в отдельных точках меридиана Солнца яркости $I_{1}$ и $I_{2}$ по величине можно считать приблизительно равными. В среднем для рассмотренных нами значений $\lambda$ они получились следуюшими:

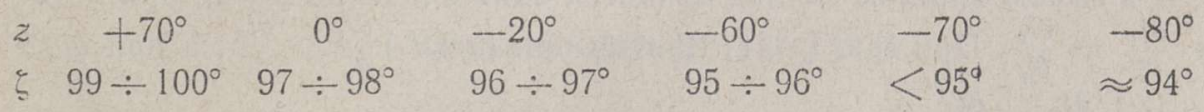

Приведенные в таблице значения ६ следует понимать как чисто ориентировочные, причем они непременно изменяются в не слишком больших пределах в зависимости от длины волны и оптических условий в атмоcфере. 
Из рис. 1а видно, что отношение $I_{2} / I_{1}$ в направлении $z=+70^{\circ}$ не увеличивается монотонно с ростом зенитного расстояния Солнца, а почти всегда имеет минимум при некоторой определенной глубине Солнца $\left(\zeta \approx 97^{\circ}\right)$. Так как аналогичный эффект наблюдается иногда и в направлении зенита, то вряд ли можно это считать просто недостатком метода, хотя на первый взгляд кажется, что отношение $I_{2} / I_{1}$ должно монотонно увеличиваться с ростом глубины Солнца.

Рассмотрим подробно упомянутый эффект. Пусть минимум отношения $I_{2} / I_{1}$ наблюдается при зенитном расстоянии Солнца $\zeta=\zeta_{0}$. Нужно отметить, что в рассмотренной нами области спектра всегда выполняется условие

$$
I_{2} /\left.I_{1}\right|_{\zeta=\zeta_{0}}<1
$$

1. В точке минимума имеем:

$$
\frac{\partial}{\partial \zeta}\left[\frac{I_{2}}{I_{1}}\right]_{\zeta=\zeta_{0}}=0, \quad \text { т. e. } \quad I_{1} \frac{\partial I_{2}}{\partial \zeta}=I_{2} \frac{\partial I_{1}}{\partial \zeta}
$$

Учитывая (6) и то, что в сумерках всегда

$$
\frac{\partial I_{2}}{\partial \zeta}<0 \quad \text { и } \quad \frac{\partial I_{1}}{\partial \zeta}<0
$$

получим из (7) $\frac{\partial I_{1}}{\partial \zeta}<\frac{\partial I_{2}}{\partial \zeta}$, т. е. при $\zeta=\zeta_{о}$ яркость первичного рассеяния убывает быстрее яркости кратного рассеяния.

2. При $\zeta<\zeta_{0}$ и $I_{2} \geqslant I_{1}$ имеем

$$
\frac{\partial}{\partial \zeta}\left[\frac{I_{2}}{I_{1}}\right]<0, \quad \text { T. e. } \quad I_{1} \frac{\partial I_{3}}{\partial \zeta}<I_{2} \frac{\partial I_{1}}{\partial \zeta}
$$

откуда, учитывая (8), следует, что $\frac{\partial I_{2}}{\partial \zeta}<\frac{\partial I_{1}}{\partial \zeta}$, т. е. $I_{2}$ убывает быстрее $I_{1}$

3. Сравнить непосредственно величины $\frac{\partial I_{2}}{\partial \zeta}$ и $\frac{\partial I_{1}}{\partial \zeta}$ при $\zeta<\zeta_{0}$ и $I_{2}<I_{1}$ по данной методике невозможно, и вопрос остается пока нерешенным.

4. В случае $\zeta>\zeta_{0}$ и $I_{2} \leqslant I_{1}$ получим $\frac{\partial I_{1}}{\partial \zeta}<\frac{\partial I_{2}}{\partial \zeta}$, т. е. $I_{1}$ убывает быстpee $I_{2}$.

5. При $\zeta>\zeta_{0}$ и $I_{2}>I_{1}$ соотношение величин $\frac{\partial I_{1}}{\partial \zeta}$ и $\frac{\partial I_{2}}{\partial \zeta}$ остается пока также неопределенным.

Из вышеизложенного можно сделать заключение, что при $\zeta<\zeta_{0}$ г1 $I_{2}<I_{1}$ в самом простом случае найдется одно значение $\zeta=\zeta_{1}$, при котором $\frac{\partial I_{1}}{\partial \zeta}=\frac{\partial I_{2}}{\partial \zeta}$. При $\xi_{2}<\zeta<\zeta_{1}\left(\zeta_{2}<\zeta_{0}\right.$ при $\left.I_{2}=I_{1}\right) \frac{\partial I_{1}}{\partial \zeta}>\frac{\partial I_{2}}{\partial \zeta}$, а при $\zeta_{1}<$ $<\zeta<\zeta_{0} \frac{\partial I_{1}}{\partial \zeta}<\frac{\partial I_{2}}{i \zeta}$. В общем случае возможно существование нескольких значений $\zeta$, при которых $\frac{\partial I_{1}}{\partial \zeta}=\frac{\partial I_{2}}{\partial \zeta}$. Значение $\zeta_{1}$ должно зависеть от длины волны $\lambda$ и различаться для разных сумерек в зависимости от оптических условий в земной атмосфере. 
Обратимся к рис. 6, на котором представлено отношение производных от яркостей в функции от зенитного расстояния Солнца для одного числа. Рассмотренный нами случай минимума в ходе функции $I_{2} / I_{1}=$ $=g(\zeta)$ соответствует на этом рисунке кривой $1(\lambda=422$ ммк). Такой эффект наблюдался всегда в направлении $z=+70^{\circ}$ для $\lambda=422$ ммк. Для $\lambda=479$ и 574 ммк он был выражен слабее или отсутствовал совсем (напр., кривая 1 для 479 ммк).

Из приведенного рисунка (кривая 1,422 ммк) видно, что при $\zeta>\zeta_{0}$ и $I_{2}>I_{1}\left(I_{2}=I_{1}\right.$ в данном случае при $\left.\zeta=\zeta_{3} \approx 99^{\circ} 40^{\prime}\right)\left|\begin{array}{l}\frac{\partial I_{1}}{\partial \zeta} \\ \partial \xi\end{array}\right|>\left|\frac{\partial I_{2}}{\partial \zeta}\right|$, но убывание рассмотренного отношения начинается уже раньше. Значит, с некоторого значения $\xi_{0}<\zeta<\zeta_{3}$ или замедляется убывание яркости $I_{1}$, или ускоряется убывание $I_{2}$.

Можно полагать, что одной из причин описанного эффекта является растущая с увеличением глубины Солнца роль более высоких слоев атмосферы в кратном рассеянии, т. е. слой кратного рассеяния расширяется в сторону более высоких слоев атмосферы, где плотность воздуха меньше. В зените такой

Рис. 6. Относительные скорости убывания яркостей $I_{1}$ и $I_{2}$ в зависимости от $\zeta, F=$ $=\left(\partial I_{1} / \partial \zeta\right)\left(\partial I_{2} / \partial \zeta\right)-1: 1-z=+70^{\circ}$,

$$
2-z=0^{\circ} \text {. }
$$

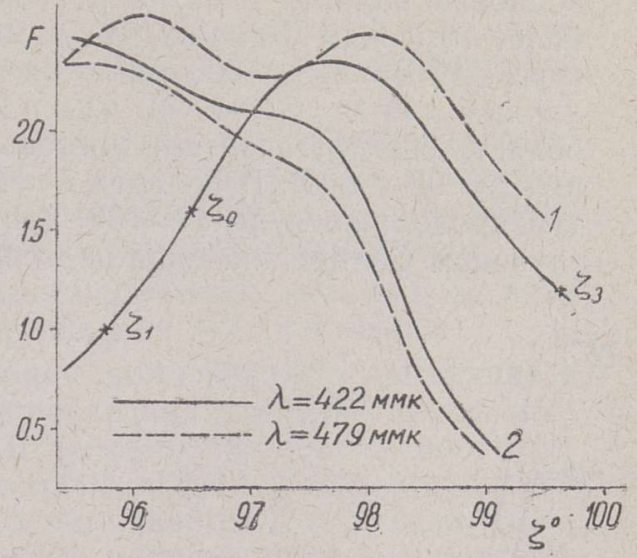

прящесс, по-видимому, начннается уже при меньших зенитных расстояЕиях Солнца. Минимумы наблюдаются также в ходе $I_{2} / I_{1}=g(\zeta)$ в зените при меньших значениях $\xi$.

Рассмотрим, чем может быть вызван эффект $\left|\frac{\partial I_{2}}{\partial \zeta}\right|>\left|\frac{\partial I_{1}}{\partial \zeta}\right|$ при $\zeta<\zeta_{1}$. Қак известно, в ранней фазе сумерек источником радиации для кратного рассеяния является весь небосвод. С ростом глубины Солнца заревой максимум яркости будет все больше доминировать над остальными участками небосвода. Можно полагать, что одной из возможных причин наблюдаемого нами эффекта при $\zeta<\zeta_{1}$ является быстрое убывание яркости $I_{2}$ за счет уменьшения размеров источника радиации. Если при этом еще учесть, что яркость первичного рассеяния $I_{1}$ убывает в зените значительно быстрее, чем в направлении $z=+70^{\circ}$, то понятно, почему наблюдаются как выравнивание величин $\frac{\partial I_{1}}{\partial \zeta}$ и $\frac{\partial I_{2}}{\partial \zeta}$, так и минимум отношения $I_{2} / I_{1}$ в зените в ранней фазе сумерек.

\section{О цвете кратного рассеяния}

Изменение спектрального состава рассеянной радиации, поступающей от сумеречного неба, отражается в исследованиях многих авторов. Характерной чертой полученных результатов является «посинение» света при малых зенитных расстояниях Солнца $\left(\zeta \geq 92^{\circ}\right)$. Зато с некоторого определенного значения $\zeta$, зависящего от зенитного расстояния 
направления визирования (в зените при $\zeta \approx 99^{\circ}$ ), во многих случаях наблюдается заметное «покраснение» сумеречного неба.

В качестве причины упомянутого покраснения предполагаются разные процессы. Одной из причин некоторые авторы считают свечение ночного неба, т. е. собственное свечение газов в верхней атмосферє под влиянием прямых лучей Солнца.

Известно, что при определенной глубине Солнца на фоне сумерешного неба появляются отдельные эмиссионные линии. Свечение ночного неба появляется сначала в длинноволновой области спектра с последующим переходом к коротким волнам [3]. Если причиной покраснения неба действительно являлось бы собственное свечение атмосферы, то следовало бы предполагать, что при рассмотренных в сумеречных исследо-

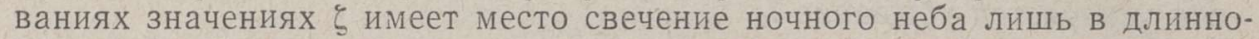
волновой области спектра и в коротковолновой оно появляется при более глубоких Солнщах. Н. Дивари $\left[{ }^{8}\right]$ определены цветовые температуры для $\lambda=370 \div 600$ ммк. Ход последних получился существенно различным для $\lambda=370 \div 440$ млк и $\lambda=440 \div 600$ ммк. В коротковолновой области спектра значения цветовых температур почти монотонно растут при $\xi=92 \div 106^{\circ}$. В области $\lambda=440 \div 600$ ммк рост цветовых температур продолжался до $\zeta \approx 100^{\circ}$ (измерения велись в зените). При более глубоком Солнце значения цветовых температур снова уменьшаются.

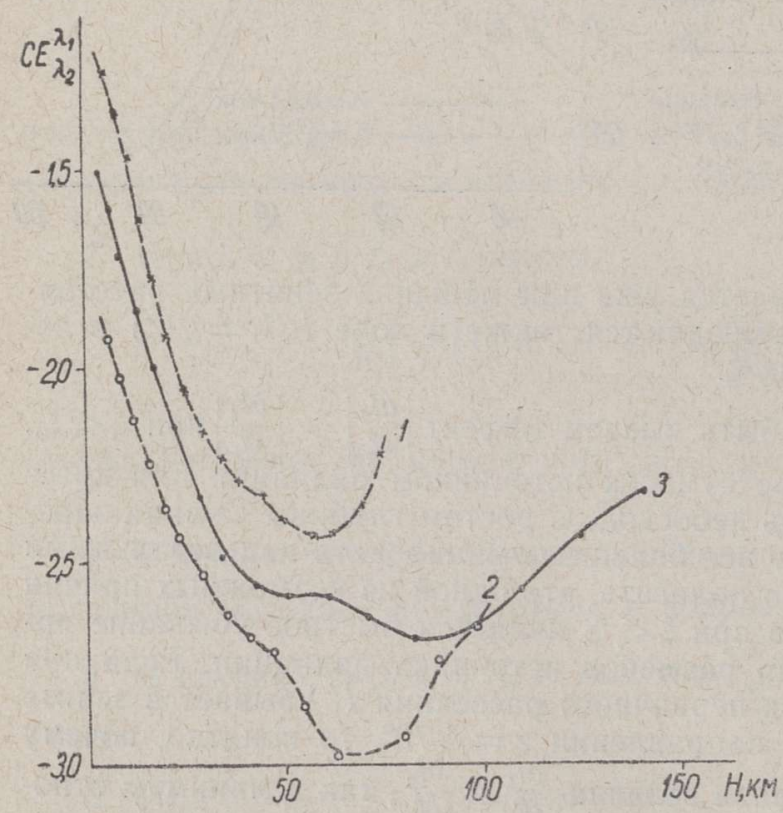

Рис. 7. Зависимость цветового показателя $C E_{\lambda_{2}}^{\lambda_{1}}$ от высоты геометрической тени Земли: $\lambda_{1} \stackrel{2}{=}$ $=422$ ммк, $\lambda_{2}=574$ м.к; $1-z=+60^{\circ}$, $2-z=0^{\circ}, 3-z=-60^{\circ}$.

Таким образом, результаты Н. Дивари показывают, что до самого наступления ночи почти не происходит изменений в спектральном составе коротковолновой области спектра.

Қроме того, если свечение ночного неба имело бы существенное влияние на цвет сумеречного неба, то эффект покраснения должен был бы наблюдаться в разных направлениях визирования при разных значениях $\xi$ (что действительно имеет место), но при одинаковых эффективных высотах земной тени (или в первом приближении при одинаковых высотах геометрической тени Земли). Однако это последнее условие не выполняется, что хорошо видно из рис. 7.

Другой возможной при чиной покраснения рассеянной радиации считается растущее влияние кратного рассеяния с увеличением зенитного расстояния Солнца в образовании общей яркости.

Согласно теории Релея, коэффициент рассеяния для газов вне полос поглощения пропорционален $\lambda^{-4}$. Следовательно, спектральная яркость $k$-кратно рассеянного света должна быть пропорциональна $\lambda^{-4 k}$. Однако, так как реальная атмосфера отличается от релеевской атмосферы и 
реальный коэффициент рассеяния пропорционален $\lambda^{-n}$, где $n<4$, то для $k$-кратного рассеяния получим зависимость вида $\lambda^{-k n}$.

Ф. Линк [9], оценивая спектральную зависимость отношения $I_{2} / I_{1}$, пришел к заключению, что оно должно быстро убывать с увеличением длины волны.

$Ф$. Юдалевичем $\left[{ }^{10}\right]$ рассчитана спектральная зависимость $I_{2} / I_{1}$ на основе соотношения

$$
\frac{\left(I_{2} / I_{1}\right)_{\lambda=\lambda_{1}}}{\left(I_{2} / I_{1}\right)_{\lambda=\lambda_{2}}} \approx\left(\frac{\lambda_{2}}{\lambda_{1}}\right)^{4}
$$

Полученные результаты позволили сделать заключение, что в ближней инфракрасной области спектра отношение $I_{2} / I_{1}$ исчисляется при любых значениях $\zeta$ лишь несколькими процентами.

Все эти результаты свидетельствуют о том, что кратное рассеяние должно привести к увеличению относительной доли коротковолновой области спектра в общей яркости.

Однако Г. Розенбергом указано на возможность обратного спектрального хода [3]. А именно, вследствие сильной экстинкции в коротковолновой области сгектра многократно рассеянный свет по своему спектральному составу может оказаться краснее первично рассеянного.

Использование данных спектрофотометрирования сумеречного неба позволило оценить спектральный ход отношения $I_{2} / I_{1}$ в сумерках.

Қак было указано выше, относительная доля кратного рассеяния в обшей яркости сумеречного неба в направлениях $z= \pm 70^{\circ}$ всегда возрастает с убыванием длины волны $\lambda$. В направлении зенита, наоборот, в большинстве случаев наблюдался обратный эффект. Такие результаты позволяют думать, что спектральный состав яркостей $I_{1}$ и $I_{2}$ (или одной из них) существенно различен в различных направлениях визирования. Это, по-видимому, обусловлено, с одной стороны, увеличением экстинкции преимущественно в коротковолновой области спектра, а с другой сильным поглощением радиации озоном в полосе Шапюи.

На основе экспериментальных данных по формулам (3), (2) и (5) определены яркости $I_{1}=I_{1}(\zeta)$ и $I_{2}=I_{2}(\zeta)$ для зенита и в направлении $z=+70^{\circ}$. Цветовые показатели $C E_{i_{2}}^{\lambda_{1}}$ вычислены по формулам

$$
C E_{\lambda_{2}}^{\lambda_{1}}\left(I_{1}\right)=-1,08 \ln \frac{I_{1}\left(\lambda_{1}\right)}{I_{1}\left(\lambda_{2}\right)} \quad \text { и } \quad C E_{\lambda_{3}}^{\lambda_{1}}\left(I_{2}\right)=-1,08 \ln \frac{I_{2}\left(\lambda_{1}\right)}{I_{2}\left(\lambda_{2}\right)}
$$

где $\lambda_{1}<\lambda_{2}$.

Рис. 8 представляет полученные результаты. Так как в ходе $C E_{\lambda_{2}}^{\lambda_{1}}$ для $\lambda_{2}=479$ ммк и $\lambda_{2}=574$ ммк (всегда $\lambda_{1}=422$ ммк) наблюдалось существенное различие, то оба эти случая представлены отдельно. Ход функции $C E_{\lambda_{2}}^{\lambda_{1}}=f(\zeta)$ для различных чисел в одной и той же спектральной области носит принципиально такоӥ же характер.

Рассмотрим сначала случай, когда $\lambda_{1}=422$ ммк и $\lambda_{2}=479$ ммк. Значения $C E_{\lambda_{2}}^{\lambda_{1}}\left(I_{1}\right)$ и $C E_{\lambda_{2}}^{\lambda_{1}}\left(I_{2}\right)$ как для зенита, так и для направления $z=$ $=+70^{\circ}$ в рассматриваемой фазе сумерек относительно мало изменяют. ся с ростом глубины Солнца, причем наблюдаемые колебания носят явно случайный характер. Во всех рассмотренных случаях яркость $I_{1}$ в зените краснее яркости $I_{2} ;$ в направлении $z=+70^{\circ}$ наблюдается обратная спектральная зависимость. В ходе цветового показателя для общей яркости $I=I_{1}+I_{2}$ также отсутствуют существенные закономерные изменения. 


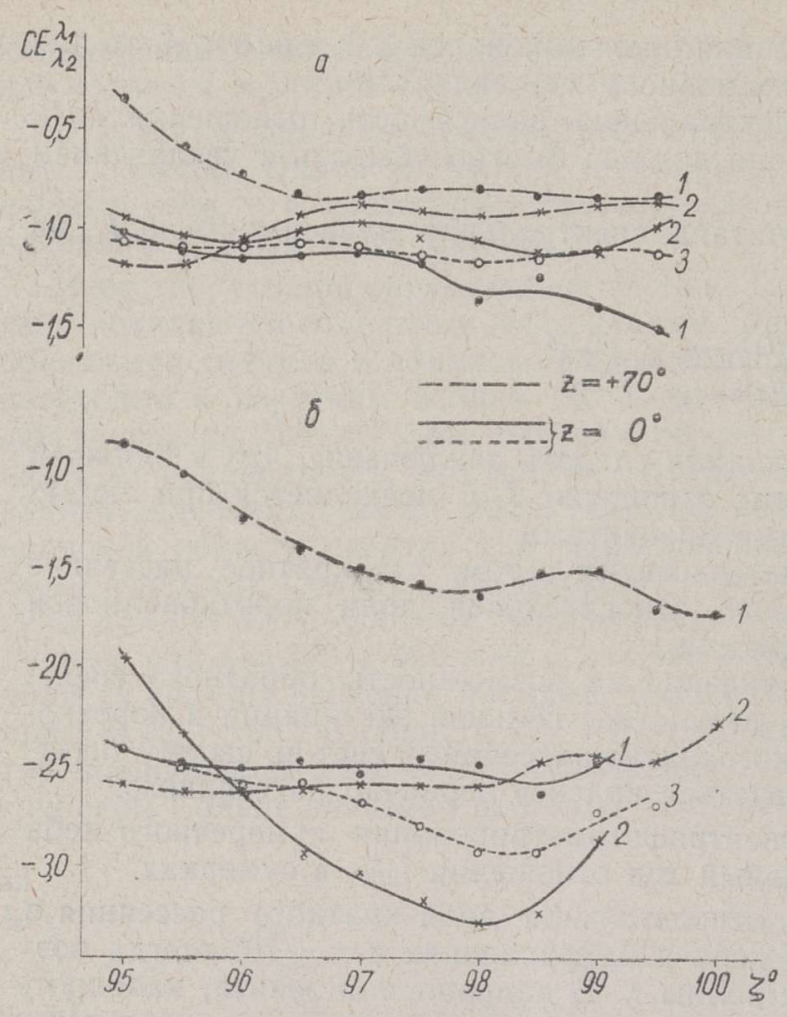

Рис. 8. Зависимости $C E_{\lambda_{-2}}^{\lambda_{1}}$ для $I_{1}, I_{2}$ и $I$ or зенитного расстояния Солнца: $1-C E_{\lambda_{2}}^{\lambda_{1}}\left(I_{1}\right), 2-C E_{\lambda_{2}}^{\lambda_{1}}\left(I_{2}\right)$, $3-C E_{\lambda_{2}}^{\lambda_{1}}(I) ; a-\lambda_{1}=422 м \mu \kappa, \lambda_{2}=479$ ммк; б- $\lambda_{1}=422$ мик, $\lambda_{2}=574$ ммк.
В области спектра $\lambda=$ $=479 \div 574$ млк, которую характеризуют цветовые показатели при $\lambda_{2}=574 м \mu \kappa$, первйчно рассеянный свет в направлении визирования $z=+70^{\circ}$ по своему спектральному составу всегда остается более красным, чем вторично рассеянный. В направлении зенита либо $I_{2}$ краснее $I_{1}$ в течение всей рассмотренной нами фазы сумерек, либо он краснее при малых значениях , но при больших значениях б, наоборот, более синий. В от личие от области $\lambda=422 \div$ $\div 479$ ммк цветовой показатель для общей яркости I имеет здесь уже определен ный ход с ростом зенитного расстояния Солнца (см. рис. 8). При малых глубинах Солнца он имитирует ход $\mathrm{CE}_{\lambda_{2}}^{\lambda_{1}}\left(I_{1}\right)=f(\zeta)$, но с уве. личением $\zeta$ все больше приближается к ходу $C E_{\lambda_{2}}^{\lambda_{1}}\left(I_{2}\right)=$ $=f(\zeta)$. Такой эффект, повидимому, отражает те особенности, которые имеют место при рассеянии света атмосферой в сумеречных условиях, когда доминируюшая роль переходит от первичного к кратному рассеянию.

С увеличением $\zeta$ величина $C E_{\lambda_{2}}^{\lambda_{1}}\left(I_{1}\right)$ изменяется слабо в сторону посинения. Зато $C E_{\lambda_{2}}^{\lambda_{1}}\left(I_{2}\right)$ существенно изменяет свою форму при малых зенитных расстояниях Солнца в направлении посинения, но, начиная с некоторого определенного значения $\zeta$ (в нашем случае оно соответствует в зените $\zeta \approx 98 \div 99^{\circ}$ ), имеет место покраснение вторично рассеянного света.

Из сказанного вытекает, что в общем случае в сумерках нет оснований считать $I_{2}$ более красным или более синим, чем $I_{1}$. Преобладание какой-нибудь области спектра определяется конкретными условиями и должно быть рассмотрено каждый раз отдельно.

Приступим к выяснению возможных причин описанного хода $C E_{\lambda_{2}}^{\lambda_{1}}\left(I_{2}\right)=f(\zeta)$. Последний наблюдается, по нашим данным, лишь при $\lambda_{2}=574$ ммк, поэтому можно полагать, что он вызывается существованием слоя озона на высоте около 25 км над земной поверхностью.

Обратимся к рис. 9. Рассмотрим сначала случай, когда Ђ мала. Тогда заревые максимумы яркости располагаются относительно высоко над горизонтом и вторичное рассеяние происходит главным образом в слое, примыкающем к земной поверхности. Предположим, что основ- 
ная часть первичного рассеянного света поступает на слой вторичного рассеяния от заревого максимума.

Пусть $z=0^{\circ}$ и вторичное рассеяние происходит в точке $A$. Первично рассеянный свет пройдет слой озона. Ослабление яркости должно быть больше при длине волны $\lambda=574$ ммк за счет поглощения озоном. К этому прнбавляется еще рост воздушной массы $m$ с увеличением $\lambda$, ибо высота заревых максимумов яркости над горизонтом уменьшается в направлении длинных волн. Если учесть еще, что рост-глубины Солнца прино-

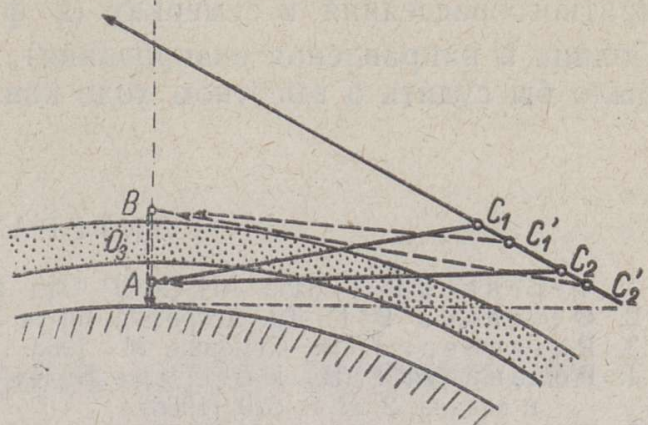

Рис. 9. К объяснению изменения цвета сумеречного неба. сит с собой приближение заре-

вых максимумов к горизонту, то нетрудно понять, что это должно вести к-посинению вторично рассеянного света, что на самом деле и наблюдалось до некоторого определенного значения $\zeta$.

Как известно [11], с ростом $\zeta$ все более высокие слои атмосферы начинают принимать участие в кратном рассеянии. Рассмотрим случай, когда вторичное рассеяние происходит над слоем озона (в точке $B$ ). В таком случае влияние $\mathrm{O}_{3}$ выявляется уже не при первично, а при вторично рассеянном свете. Здесь также значительно больше ослабляется радиация в области $\lambda=574$ ммк, но путь луча после вторичного рассеяния уже одинаков для разных длин волн и поглощение радиации $I_{2}(\lambda=$ $=574$ ммк) в слое озона не изменяется с ростом $\zeta$.

В направлении $z=+70^{\circ}$ имеют место в принципе аналогичные процессы, хотя здесь диапазон изменений $C E_{\lambda_{2}}^{\lambda_{1}}\left(I_{2}\right)$ меньше. Это вполне понятно, если учесть, что относительные изменения длины пути луча в слое $\mathrm{O}_{3}$ с ростом $\zeta$ в зените больше, чем в направлении $z=+70^{\circ}$.

Покраснение кратно рассеянного света $I_{2}$ в направлении $z=+70^{\circ}$ наблюдается немного позже, чем в зените. Это свидетельствует о том, что скорость поднятия слоя кратного рассеяния в разных направлениях визирования различна.

Резюмируя вышеизложенное, можно сказать, что существенную роль в изменении цвета сумеречного неба играет атмосферный озон (см. также [8]). Посинение сумеречного неба при малых $\zeta$ обусловлено, с одной стороны, посинением первично рассеянного света за счет уменьшения $q=-\frac{d \ln I_{1}}{d H} \quad$ с ростом высоты геометрической тени Земли $H\left[{ }^{3}\right]$, а с другой стороны, за счет возрастания влияния кратно рассеянной радиации $I_{2}$, которая по своему спектральному составу становится все более синей, пока слой кратного рассеяния не поднимется достаточно высоко в атмосферу. Покраснение неба наблюдается с момента, когда в кратном рассеянии начинают принимать участие и слои атмосферы, где поглощение озоном незначительно. Так как к этому времени $I_{2}>I_{1}$, то покраснение кратно рассеянной радиации $I_{2}$ главным образом и определяет спектральный состав общей яркости неба.

Одновременное определение высотного хода плотности озона и $C E_{\lambda_{2}}^{\lambda_{1}}\left(I_{2}\right)=f(\zeta)$ при значении $\lambda_{2}$ в полосе поглощения Шапюи позволило бы оценить в разных фазах сумерек те высоты, которые преимущественно ответственны за кратное рассеяние. 
Кроме того, если каким-нибудь методом удалось бы из данных спектрофотометрирования сумеречного неба определить высоту слоя кратного рассеяния в сумерках (в функции от зенитного расстояния Солнца и направления визирования), то по ходу $C E_{\lambda_{2}}^{\lambda_{1}}\left(I_{2}\right)=f(\zeta)$ можно было бы судить о высотном ходе концентрации озона.

\section{ЛИТЕРАТ У РА}

1. Фесенков В. Г., Изв. АН СССР. Отд. матем. и естеств. наук, 1501 (1934)

2. Фесенков В. Г., Тр. Астрофиз. ин-та АН Каз. ССР, 3, 214 (1962).

3. Р озенбе рг Г. В., Сумерки, М., 1963.

4. Р оз ен бе рг Г. В., Пылд м а а В. Қ., Изв. АН СССР. Сер. Физика атмосферы и океана, 2, № 8, 820 (1966).

5. П ылд м а а В., Изв. АН ЭССР. Сер. физ.-матем. и техн. наук, 13, № 3, 192 (1964)

6. Ди в а р и Н. Б., Плотников а Л. И., Астрон. Ж., 42, № 5, 1090 (1965).

7. П ыл д м а В В. К., Изв. АН СССР. Сер. Физика атмосферы и океана, 1, № 11 , 1168 (1965)

8. Д и в а р и Н. Б., ДАН СССР, 122, № 5,795 (1958).

9. L i n k F., Bull. Astron. Inst. Czechosl., 1, No. 9, 135 (1949)

10. Юд а л е в и ч Ф. Ф., Изв. АН СССР. Сер. геофиз., № 7, 862 (1956)

11. D a v e J. V., Proc. Ind. Acad. Sci., 43, Sec. A, No. 6, 336 (1956).

Ннститут физики и астрономии Академии наук Эстонской ССР
Поступила в редакцию 2/III 1966

\section{POLDMAA}

\section{KORGEMAT JÄRKU HAJUMISE OSATÄHTSUSEST HÄMARIKUS}

Töös vaadeldakse kõrgemat järku hajumise mõju hämarikutaeva heledusele. Taeva absoluutseid heledusi on autor mõõtnud kolmes kitsas spektraalpiirkonnas $(\lambda=422,479$ ja $574 \mathrm{mu}$ ) 1963. aastal. Kōrgemat järku hajumise osa taeva koguheleduses on leitud Päikese meridiaanil kolmes suunas: seniidis G. Rosenbergi ja suundades $z= \pm 70^{\circ}$ täpsustatud V. Fessenkovi meetodil. Vaadeldakse esimest ja kõrgemat järku hajumisest tingitud taeva heleduste $I_{1}$ ja $I_{2}$ muutusi Päikese seniitkauguse $\zeta$ funktsioonina. Analüüsitakse suhte $I_{2} / I_{1}$ muutumise iseärasusi hämariku eri faasides.

Töö teises osas võrreldakse heledusi $I_{1}$ ja $I_{2}$ spektri mitmes piirkonnas Päikese erinevatel seniitkaugustel. Analüüsitakse värvusindeksi olenevust $\zeta$-st ja näidatakse, et hämarikutaeva hajusa kiirguse spektraalse koostise muutusi põhjustab osooni kiirgust neelava toime ja kõrgemat järku hajumise kihi kõrguse muutuse koosmõju.

\section{POLDMAA}

\section{ON HIGH-ORDER SCATTERING IN TWILIGHT}

The report deals with the influence of the high-order scattering on the brightness of the twilight sky. The brightness of the sky in absolute units of energy was measured in three narrow spectral ranges $(\lambda=422 ; 479 ; 574 \mathrm{~m} \mu)$ by the author in 1963 . The role of the whole brightness of the sky is found on the Sun's vertical in three directions: in the zenith by the G. Rosenberg's method and in directions $z= \pm 70^{\circ}$ by the precisely defined V. Fesenkov's method. The changes in sky brightness caused by the first - $\left(I_{1}\right)$ and high-order $\left(I_{2}\right)$ scattering as a function of the Sun's zenith distance $\zeta$ are examined. The peculiarities of the change of the relation $I_{2} / I_{1}$ in different phases of twilight are analysed.

In the second part of this report the brightnesses $I_{1}$ and $I_{2}$ in different regions of the spectrum for different zenith distances of the Sun are compared. The dependence of the colour index on $\xi$ is analysed. It is also shown that changes in the spectral composition of the diffuse radiation of the twilight sky are caused by the co-operation of the radiation-absorbing influence of ozone and of the alteration of the height of the highorder scattering layer. 